Running head: SUPPLEMENTARY MATERIAL FOR CHATTOPADHYAY AND KELLEY

\author{
Supplementary Material for \\ "Estimation of the Coefficient of Variation with Minimum Risk: A Sequential Method for \\ Minimizing Sampling Error and Study Cost" \\ (Multivariate Behavioral Research, X, X-X)
}

\author{
Bhargab Chattopadhyay \\ Department of Mathematical Sciences \\ University of Texas at Dallas \\ Richardson, Texas USA
}

Ken Kelley

Department of Management

University of Notre Dame

Notre Dame, Indiana USA 


\section{Supplementary Material for \\ "Estimation of the Coefficient of Variation with Minimum \\ Risk: A Sequential Method for Minimizing Sampling Error and \\ Study Cost"}

(Multivariate Behavioral Research, $\mathrm{X}, \mathrm{X}-\mathrm{X}$ )

In this supplementary material, we provide different mathematical results that are associated with our proposed sequential procedure and also the simulation study showing thhe neglibility of the remainder term of the MSE of $k_{n}$ for not too small sample sizes.

\section{Theoretical Results}

In this subsection, we provide a proof that if observations are collected using Equation 25 of Chattopadhyay and Kelley (in press), then sampling will stop at some stage of the sequential procedure with probability one. This is proved in Lemma 1, which shows that, under appropriate conditions, $P\left(N_{c}<\infty\right)=1$. This result is very important, as it ensures mathematically that the sequential sampling procedure we discuss will be terminated eventually.

Lemma 1. Under the assumption that $\xi<\infty$, for any $c>0$, the stopping time $N_{c}$ is finite, that is, $P\left(N_{c}<\infty\right)=1$.

Proof. Note that $V_{n}$ is a strongly consistent estimator of $\xi$. Hence, the result can be obtained from the fact that $V_{n} \rightarrow \xi$ almost surely as $n \rightarrow \infty$.

The estimator $k_{N_{c}}$ is the asymptotically minimum risk point estimator (AMRPE) if the ratio of the risk function for $k_{N_{c}}$ (defined in Equation 23) and to the minimum asymptotic risk (defined in Equation 12) is asymptotically 1. Mathematically, the estimator $k_{N_{c}}$ is AMRPE if

$$
\lim _{c \downarrow 0} R_{N_{c}}(\kappa) / R_{n_{c}}(\kappa)=1 .
$$


For more details, please refer to Sen (1981). In our notation $c \downarrow 0$ means " $c$ converges to a small positive number greater than 0". That is, $c$ is always positive and cannot take the value of exactly 0 .

We formally state the properties enjoyed by our purely sequential procedure in the following theorem:

Theorem 1. If the parent distribution $F$ is such that $\mathrm{E}\left[X^{16}\right]$ and $\mathrm{E}\left[X^{-24}\right]$ exist, then the stopping rule, that is Equation 21, yields the following asymptotic optimality properties: Part 1. $\mathrm{E}\left(N_{c} / n_{c}\right) \rightarrow 1$ as $c \downarrow 0$.

Part 2. If $\gamma \in\left(0, \frac{1}{2}\right), R_{N_{c}}(\kappa) / R_{n_{c}}(\kappa) \rightarrow 1$ as $c \downarrow 0$.

Part 1 of this theorem implies that, on average, the final sample size of our procedure is asymptotically the same as the optimal sample size, $n_{c}$. Part 2 proves that the ratio regret is asymptotically 1 as in Equation 24 which means, on an average, the risk, $R_{N_{c}}(\kappa)$, at the final sample size, $N_{c}$, is close to the minimized risk, $R_{n_{c}}(\kappa)$. Thus, $k_{N_{c}}$ is the asymptotically minimum risk point estimator of $\kappa$. The properties mentioned in Parts 1 and 2 are known as asymptotic first-order efficiency and asymptotic first-order risk efficiency, respectively.

Proof. We note that the stopping rule, $N_{c}$ is as follows:

$$
N_{c} \text { is the smallest integer } n(\geq m) \text { such that } n^{2} \geq \frac{A}{c}\left(V_{n}^{2}+n^{-2 \gamma}\right) \text {, }
$$

From Equation 25 that $N_{c} \geq \sqrt{\frac{A}{c}} N_{c}^{-\gamma}$, that is, $N_{c} \geq\left(\frac{A}{c}\right)^{\frac{1}{2(1+\gamma)}}$ with probability 1 . For fixed $\epsilon, \gamma>0$, note the following definitions:

$$
n_{1 c}=\left(\frac{A}{c}\right)^{\frac{1}{2(1+\gamma)}}, \quad n_{2 c}=n_{c}(1-\epsilon), \quad \text { and } n_{3 c}=n_{c}(1+\epsilon), n_{c}=\sqrt{\frac{A}{c}} \xi
$$

One interesting property of U-statistics is that they are reverse submartingales (refer to Sen, 1981, p. 50). ${ }^{1}$ For details about reverse submartinagles and their properties, we refer to classic textbooks on probability theory and stochastic processes such as Loève (1963), 
Doob (1953) and others. Using several results of Sen and Ghosh (1981), Sen (1981), Lee (1990), Ghosh et al. (1997), Gut (2009) and De and Chattopadhyay (2015) and also using properties of U-statistics we prove the following lemmas to prove the main theorem.

From Jeffreys and Jeffreys (1988), we know a function $g(x)$ satisfies the Lipschitz condition of order $\alpha$ at $x=0$ if

$$
|g(x)-g(0)| \leq M|x|^{\alpha}
$$

for all $|x|<\epsilon$, where $M$ and $\alpha$ are independent of $x, \alpha>0$, and $\mathrm{M}$ is an upper bound for all $\alpha$ for which a finite $\mathrm{M}$ exists.

Lemma 2. Suppose $U_{n}$ is a U-statistic of degree $v$ for estimating $\theta$. Suppose, $h^{\prime}(\theta) \neq 0$ and the second derivative $h "(x)$ satisfies the Lipschitz condition. Then for positive integers $r$ we have,

$$
\mathrm{E}\left[h\left(U_{n}\right)-h(\theta)\right]^{r}=O\left(n^{-r / 2}\right) .
$$

Proof. Applying Taylor's theorem,

$$
h\left(U_{n}\right)-h(\theta)=\left(U_{n}-\theta\right) h^{\prime}(\theta)+\frac{1}{2}\left(U_{n}-\theta\right)^{2} h^{\prime \prime}(\theta)+H_{n 1}
$$

where, $H_{n 1}=\frac{1}{2}\left(U_{n}-\theta\right)^{2}\left(h "(\theta)+\lambda_{n}\left(\left(U_{n}-\theta\right)-h^{\prime \prime}(\theta)\right)\right.$.

Using the Lipschitz condition and taking expectations of both sides, incorporating Sen (p. 55, 1981), we have,

$$
\left.\mathrm{E}\left[h\left(U_{n}\right)-h(\theta)\right]^{r}=O\left(n^{-r / 2}\right)\right) .
$$

Lemma 3. Suppose that nonnegative i.i.d. observations $X_{1}, \ldots, X_{n}$ are such that $E\left(X_{1}^{4 r}\right)$ 
and $E\left(X_{1}^{-6 r}\right)$ exist for some $r \geq 1$. For any $\epsilon \in(0,1)$ and $\gamma>0$,

Part $1 P\left(N_{c} \leq n_{c}(1-\epsilon)\right)=O\left(n_{1 c}^{-\frac{r}{2}}\right)=O\left(c^{\frac{r}{4(1+\gamma)}}\right)$ as $c \downarrow 0$,

Part $2 P\left(N_{c} \geq n_{c}(1+\epsilon)\right)=O\left(n_{1 c}^{-\frac{r}{2}}\right)=O\left(c^{\frac{r}{(1+\gamma)}}\right)$ as $c \downarrow 0$.

Proof. Using the definition of stopping rule $N_{c}$ in Equation 21 and Equation 25, we have

$$
\begin{aligned}
P\left(N_{c} \leq n_{2 c}\right) & \leq P\left(n>\sqrt{\frac{A}{c}} V_{n} \text { for some } n \in\left[n_{1 c}, n_{2 c}\right]\right) \\
& \leq P\left(V_{n}^{2} \leq\left(\frac{c}{A}\right) n_{2 c}^{2} \text { for some } n \in\left[n_{1 c}, n_{2 c}\right]\right) \\
& \leq P\left(\left|V_{n}^{2}-\xi^{2}\right| \geq \xi^{2} \epsilon(2-\epsilon) \text { for some } n \in\left[n_{1 c}, n_{2 c}\right]\right) \\
& \leq P\left(\max _{n_{1 c} \leq n \leq n_{2 c}}\left\{\left|V_{1 n}\right|+\left|V_{2 n}\right|+\left|V_{3 n}\right|+\left|V_{4 n}\right|\right\} \geq \xi^{2} \epsilon(2-\epsilon)\right),
\end{aligned}
$$

where $V_{1 n}=\left(\frac{\widehat{\mu}_{4 n}}{4 X_{n}^{4}}-\frac{\mu_{4}}{4 \mu^{4}}\right), V_{2 n}=\left(\frac{\widehat{\mu}_{3 n}}{X_{n}^{3}}-\frac{\mu_{3}}{\mu^{3}}\right), V_{3 n}=\left(\frac{s_{n}^{4}}{4 X_{n}^{4}}-\frac{\sigma^{4}}{4 \mu^{4}}\right)$, and $V_{4 n}=\left(\frac{s_{n}^{2}}{2 X_{n}^{2}}-\frac{\sigma^{2}}{2 \mu^{2}}\right)$. Let $k=\xi^{2} \epsilon(2-\epsilon)$. Then, Equation 28 can be written as $P\left(N_{c} \leq n_{2 c}\right) \leq P_{1}+P_{2}+P_{3}+P_{4}$, where

$$
P_{i}=P\left(\max _{n_{1 c} \leq n \leq n_{2 c}}\left|V_{i n}\right| \geq \frac{k}{4}\right), \quad \text { for } i=1,2,3,4
$$

First, let us find an upper bound of $P_{1}$. Let $T_{1 n}=\left(\frac{1}{\bar{X}_{n}^{2 p}}-\frac{1}{\mu^{2 p}}\right), T_{2 n}=\widehat{\mu}_{4 n}-\mu_{4}$. Note that

$$
V_{1 n}=T_{1 n} T_{2 n}+\mu_{4} T_{1 n}+\frac{1}{\mu^{4}} T_{2 n}, \text { for } p=2
$$

Let us consider the first term in the summation of Equation 30. Using lemma 2, maximal inequality for reverse martingales Lee (1990, p.112), lemma 2.2 of Sen and Ghosh (1981), and lemma 7.3 of De and Chattopadhyay (2015), we have,

$$
\begin{aligned}
P\left(\max _{n_{1 c} \leq n \leq n_{2 c}}\left|T_{1 n} T_{2 n}\right| \geq \frac{k}{3}\right) & \leq \sum_{i=1}^{2} P\left(\max _{n_{1 c} \leq n \leq n_{2 c}}\left|T_{i n}\right| \geq\left(\frac{k}{3}\right)^{\frac{1}{2}}\right) \\
& \leq O\left(n_{1 c}^{-r}\right)+O\left(n_{1 c}^{-r / 2}\right)=O\left(n_{1 c}^{-r / 2}\right) .
\end{aligned}
$$


Following the same argument as above, one can show that the asymptotic order of probability of remaining terms in the summation of Equation 30 are either $O\left(n_{1 c}^{-r / 2}\right)$ or $O\left(n_{1 c}^{-r}\right)$. Therefore,

$$
P_{1}=P\left(\max _{n_{1 c} \leq n \leq n_{2 c}}\left|V_{1 n}\right| \geq \frac{k}{4}\right) \leq O\left(n_{1 c}^{-r / 2}\right)
$$

Note that all of the estimators in $V_{2 n}, V_{3 n}$ and $V_{4 n}$ are based on the ratio of the U-statistics to the sample mean. The ratio is comparable to what we had in the case of $V_{1 n}$. So, proceeding along the same lines as in the proof of Equation 32, one can show that $P_{2}, P_{3}$ and $P_{4}$ are $O\left(n_{1 c}^{-r / 2}\right)$ as $c \downarrow 0$. We complete the proof of Part 1 by adding all the upper bounds for $P_{1}-P_{4}$ and noting that $n_{1 c}=O\left(c^{-1 /(2+2 \gamma)}\right)$. The proof for Part 2 of lemma 3 is very similar to the proof of Part 1.

Lemma 4. If nonnegative i.i.d. observations $X_{1}, \ldots, X_{n}$ are such that $\mathrm{E}\left[X_{1}^{4 r}\right]$ and $\mathrm{E}\left[X_{1}^{-4 r \alpha}\right]$ exist for some $r \geq 1$ and $\alpha>1$, then

$$
\mathrm{E}\left[\max _{n_{1 c} \leq n \leq n_{2 c}}\left(k_{n}-\kappa\right)^{r}\right]=O\left(n_{1 c}^{-r / 2}\right) \text { as } c \downarrow 0 .
$$

Proof. Applying $C_{r}$ inequality, we can write

$$
\begin{aligned}
\left(k_{n}-\kappa\right)^{r} & =\left\{s_{n}\left(\frac{1}{\bar{X}_{n}}-\frac{1}{\mu}\right)+\frac{1}{\mu}\left(s_{n}-\sigma\right)\right\}^{r} \\
& \leq \frac{1}{2}\left\{s_{n}^{r}\left(\frac{1}{\bar{X}_{n}}-\frac{1}{\mu}\right)^{r}+\frac{1}{\mu^{r}}\left(s_{n}-\sigma\right)^{r}\right\}
\end{aligned}
$$


By the Cauchy-Schwarz inequality and Lemma 9.2.4 of Ghosh et al. (1997), we have

$$
\begin{aligned}
& 2 \mathrm{E}\left[\max _{n_{1 c} \leq n \leq n_{2 c}}\left(k_{n}-\kappa\right)^{r}\right] \\
& \leq\left\{\mathrm{E}\left[\max _{n_{1 c} \leq n \leq n_{2 c}} s_{n}^{2 r}\right] \mathrm{E}\left[\max _{n_{1 c} \leq n \leq n_{2 c}}\left(\frac{1}{\bar{X}_{n}}-\frac{1}{\mu}\right)^{2 r}\right]\right\}^{\frac{1}{2}}+\frac{1}{\mu^{r}}\left(\frac{r}{r-1}\right)^{r} \mathrm{E}\left[s_{n_{1 c}}-\sigma\right]^{r} \\
& \leq\left\{\mathrm{E}\left[\max _{n_{1 c} \leq n \leq n_{2 c}} s_{n}^{2 r}\right]\right\}^{\frac{1}{2}}\left\{\frac{1}{\mu^{2 r}} \mathrm{E}\left[\max _{n_{1 c} \leq n \leq n_{2 c}}\left(\bar{X}_{n}-\mu\right)^{4 r}\right] \mathrm{E}\left[\max _{n_{1 c} \leq n \leq n_{2 c}} \frac{1}{\bar{X}_{n}^{4 r}}\right]\right\}^{\frac{1}{4}}+O\left(n_{1 c}^{-r / 2}\right) .
\end{aligned}
$$

The last inequality is obtained by the Cauchy-Schwarz inequality and Lemma 2. Note that, by Lemma 9.2.4 of Ghosh et al. (1997), Lemma 2.2 of Sen and Ghosh (1981), and existence of $\mathrm{E}\left[X_{1}^{4 r}\right]$

$$
\begin{aligned}
& \mathrm{E}\left[\max _{n_{1 c} \leq n \leq n_{2 c}}\left(\bar{X}_{n}-\mu\right)^{4 r}\right] \leq\left(\frac{4 r}{4 r-1}\right)^{4 r} \mathrm{E}\left[\bar{X}_{n_{1 c}}-\mu\right]^{4 r} \leq O\left(n_{1 c}^{-2 r}\right), \\
& \mathrm{E}\left[\max _{n_{1 c} \leq n \leq n_{2 c}} s_{n}^{2 r}\right] \leq\left(\frac{2 r}{2 r-1}\right)^{2 r} \mathrm{E}\left[s_{n_{1 c}}^{2 r}\right]<\infty, \text { and } \\
& \mathrm{E}\left[\max _{n_{1 c} \leq n \leq n_{2 c}} \frac{1}{\bar{X}_{n}^{4 r}}\right] \leq 1+\int_{1}^{\infty} P\left(\max _{n_{1 c} \leq n \leq n_{2 c}} \frac{1}{\bar{X}_{n}^{4 r}} \geq t\right) d t \leq 1+\frac{\mathrm{E}\left[\bar{X}_{n_{1 c}}^{-4 r \alpha}\right]}{\alpha-1}
\end{aligned}
$$

is finite as $\mathrm{E}\left[X_{1}^{-4 r \alpha}\right]<\infty$. The last inequality is due to the maximal inequality for reverse submartingales (Lee, 1990, p. 112). Using Equation 35-Equation 37 in the upper bound for Equation 34, we complete the proof.

Lemma 5. If $\mathrm{E}\left[X_{1}^{8}\right]$ and $\mathrm{E}\left[X_{1}^{-\alpha}\right]$ exist for $\alpha>8$, then $\mathrm{E}\left[\sup _{n \geq m} V_{n}^{2}\right]<\infty$ for $m \geq 4$, where sup is an abbreviation for the supremum operator.

Proof. To prove Lemma 5, it is enough to show that:

(a) $\mathrm{E}\left[\sup _{n \geq m} \frac{s_{n}^{4}}{\bar{X}_{n}^{4}}\right]$, (b) $\mathrm{E}\left[\sup _{n \geq m}\left|\frac{\widehat{\mu}_{4 n}}{\bar{X}_{n}^{4}}\right|\right]$, (c) $\mathrm{E}\left[\sup _{n \geq m} \frac{\widehat{\mu}_{3 n}}{\bar{X}_{n}^{3}}\right]$, and (d) $\mathrm{E}\left[\sup _{n \geq m} \frac{s_{n}^{2}}{X_{n}^{2}}\right]$ are finite.

Here, we have $\mathrm{E}\left[\sup _{n \geq m} s_{n}^{4}\right]<\infty$ if $\mathrm{E}\left[X_{1}^{\alpha}\right]<\infty$ for $\alpha>4$ and $m \geq 4$. By Equation 32, 
$\mathrm{E}\left[\sup _{n \geq m} \bar{X}_{n}^{-4}\right]<\infty$ if $\mathrm{E}\left[X_{1}^{-\alpha}\right]<\infty$ for $\alpha>4$. Therefore, for $\mathrm{p}=1,2$, we have,

$$
\mathrm{E}\left[\sup _{n \geq m} s_{n}^{2 p} \bar{X}_{n}^{-2 p}\right] \leq\left\{\mathrm{E}\left[\sup _{n \geq m} s_{n}^{4 p}\right] \mathrm{E}\left[\sup _{n \geq m} \bar{X}_{n}^{-4 p}\right]\right\}^{1 / 2 p}<\infty
$$

Applying the Cauchy-Schwarz inequality twice,

$$
\mathrm{E}\left[\sup _{n \geq m}\left|\frac{\widehat{\mu}_{4 n}}{\bar{X}_{n}^{4}}\right|\right] \leq\left\{\mathrm{E}\left[\sup _{n \geq m}\left|\widehat{\mu}_{4 n}^{2}\right|\right]\right\}^{\frac{1}{2}}\left\{\mathrm{E}\left[\sup _{n \geq m}\left|\bar{X}_{n}^{-8}\right|\right]\right\}^{\frac{1}{2}}<\infty
$$

if $\mathrm{E}\left[X_{1}^{8}\right]$ and $\mathrm{E}\left[X_{1}^{-\alpha}\right]$ exist for $\alpha>8$. Also, note that $\bar{X}_{n}, s_{n}^{2}, \widehat{\mu}_{3 n}$ and $\widehat{\mu}_{4 n}$ are U-statistics of degrees 3 and 4 respectively. Using Lemma 9.2.4 of Ghosh et al. (1997), we can say

$$
\mathrm{E}\left[\sup _{n \geq m}\left|\widehat{\mu}_{i n}^{2}\right|\right] \leq 4 \mathrm{E}\left[\left|X_{m}^{2 i}\right|\right], i=3,4
$$

Similarly, we can show that $\mathrm{E}\left[\sup _{n \geq m} \frac{\widehat{\mu}_{3 n}^{2}}{\bar{X}_{n}^{6}}\right]<\infty$ if $\mathrm{E}\left[X_{1}^{6}\right]$ and $\mathrm{E}\left[X_{1}^{-\alpha}\right]$ exist for $\alpha>6$. This completes the proof.

Lemma 6. For any $\epsilon \in(0,1)$,

$$
\mathrm{E}\left[\max _{n_{2 c} \leq n \leq n_{c}}\left(s_{n}-s_{n_{c}}\right)^{4}\right]=O\left(\frac{\epsilon}{n_{c}^{2}}\right) \quad \text { as } c \downarrow \downarrow 0 .
$$

Proof. This proof is along the same lines as the proof of Lemma 7.7 of De and Chattopadhyay (2015). We note, $\left(s_{n}-s_{n_{c}}\right) \leq\left(s_{n}^{2}-s_{n_{c}}^{2}\right)$. Because $\left\{s_{n}^{2}-s_{n_{c}}^{2}\right\}_{n=n_{2 c}}^{n_{c}}$ is a reverse martingale, lemma 9.2.4 of Ghosh et al. (1997) yields

$$
\mathrm{E}\left(\max _{n_{2 c} \leq n \leq n_{c}}\left(s_{n}-s_{n_{c}}\right)^{4}\right) \leq \mathrm{E}\left(\max _{n_{2 c} \leq n \leq n_{c}}\left(s_{n}^{2}-s_{n_{c}}^{2}\right)^{4}\right) \leq\left(\frac{4}{3}\right)^{4} \mathrm{E}\left(s_{n_{2 c}}^{2}-s_{n_{c}}^{2}\right)^{4}
$$

Let $W_{n}=s_{n}^{2}-\sigma^{2}$. Using reverse martingale property of $W_{n}$, i.e., $\mathrm{E}\left(W_{n_{2 c}} \mid \mathcal{F}_{n_{c}}\right)=W_{n_{c}}$, we 
have

$$
\begin{aligned}
& \mathrm{E}\left(W_{n_{2 c}} W_{n_{c}}^{3}\right)=\mathrm{E}\left(W_{n_{c}}^{4}\right), \quad \mathrm{E}\left(W_{n_{2 c}}^{3} W_{n_{c}}\right) \geq \mathrm{E}\left(W_{n_{c}}^{4}\right), \quad \text { and } \\
& \mathrm{E}\left(W_{n_{2 c}}^{2} W_{n_{c}}^{2}\right) \leq\left\{\mathrm{E}\left(W_{n_{2 c}}^{4}\right) \mathrm{E}\left(W_{n_{c}}^{4}\right)\right\}^{\frac{1}{2}} \leq \mathrm{E}\left(W_{n_{2 c}}^{4}\right) .
\end{aligned}
$$

Using Equation 40-Equation 41 and asymptotic form of the $r^{\text {th }}$ central moment of function of U-statistics in lemma 2,

$$
\begin{aligned}
\mathrm{E}\left[\left(s_{n_{2 c}}^{2}-s_{n_{c}}^{2}\right)^{4}\right] & =\mathrm{E}\left[W_{n_{2 c}}^{4}\right]+\mathrm{E}\left[W_{n_{c}}^{4}\right]-4 \mathrm{E}\left[W_{n_{2 c}} W_{n_{c}}^{3}\right]-4 \mathrm{E}\left[W_{n_{2 c}}^{3} W_{n_{c}}\right]+6 \mathrm{E}\left[W_{n_{2 c}}^{2} W_{n_{c}}^{2}\right] \\
& \leq 7\left\{\mathrm{E}\left[W_{n_{2 c}}^{4}\right]-\mathrm{E}\left[W_{n_{c}}^{4}\right]\right\}=O\left(\frac{1}{n_{2 c}^{2}}-\frac{1}{n_{c}^{2}}\right)+o\left(\frac{1}{n_{c}^{2}}\right)=O\left(\frac{\epsilon}{n_{c}^{2}}\right) .
\end{aligned}
$$

Equation 42 is obtained by noting that $n_{2 c}=n_{c}(1-\epsilon)$. Hence, the proof is complete.

Lemma 7. If nonnegative i.i.d. observations $X_{1}, \ldots, X_{n}$ are such that $\mathrm{E}\left[X_{1}^{8}\right]$ and $\mathrm{E}\left[X_{1}^{-16 \alpha}\right]$ exist for $\alpha>1$, then for $\epsilon \in(0,1)$,

$$
\mathrm{E}\left[\max _{n_{2 c} \leq n \leq n_{3 c}}\left(k_{n}-k_{n_{c}}\right)^{2}\right]=O\left(\frac{\sqrt{\epsilon}}{n_{c}}\right) \text { as } c \downarrow 0 \text {. }
$$

Proof. $\mathrm{E}\left[\max _{n_{2 c} \leq n \leq n_{3 c}}\left(k_{n}-k_{n_{c}}\right)^{2}\right] \leq \mathrm{E}_{1}+\mathrm{E}_{2}$, where $\mathrm{E}_{2}=\mathrm{E}\left[\max _{n_{c} \leq n \leq n_{3 c}}\left(k_{n}-k_{n_{c}}\right)^{2}\right]$, and

$$
\begin{aligned}
\mathrm{E}_{1} & =\mathrm{E}\left[\max _{n_{2 c} \leq n \leq n_{c}}\left(k_{n}-k_{n_{c}}\right)^{2}\right] \\
& =\mathrm{E}\left[\max _{n_{2 c} \leq n \leq n_{c}}\left\{\left(\frac{1}{\bar{X}_{n}}-\frac{1}{\bar{X}_{n_{c}}}\right) s_{n}+\frac{1}{\bar{X}_{n_{c}}}\left(s_{n}-s_{n_{c}}\right)\right\}^{2}\right] \leq \mathrm{E}_{11}+\mathrm{E}_{12},
\end{aligned}
$$

where $\mathrm{E}_{11}=\mathrm{E}\left[\max _{n_{2 c} \leq n \leq n_{c}}\left(\frac{1}{X_{n}}-\frac{1}{X_{n_{c}}}\right)^{2} s_{n}^{2}\right]$ and $\mathrm{E}_{12}=\mathrm{E}\left[\max _{n_{2 c} \leq n \leq n_{c}} \frac{1}{X_{n_{c}}^{2}}\left(s_{n}-s_{n_{c}}\right)^{2}\right]$. Applying the Cauchy-Schwarz inequality three times, we can write

$$
\mathrm{E}_{11} \leq\left\{\mathrm{E}\left[\max _{n_{2 c} \leq n \leq n_{c}}\left(\bar{X}_{n}-\bar{X}_{n_{c}}\right)^{4}\right]\right\}^{\frac{1}{2}}\left\{\mathrm{E}\left[\max _{n_{2 c} \leq n \leq n_{c}} s_{n}^{8}\right]\right\}^{\frac{1}{4}}\left\{\mathrm{E}\left[\frac{1}{\bar{X}_{n_{c}}^{16}}\right] \mathrm{E}\left[\max _{n_{2 c} \leq n \leq n_{c}} \frac{1}{\bar{X}_{n}^{16}}\right]\right\}^{\frac{1}{8}}
$$


Using Lemma 7.7 of De and Chattopadhyay (2015), Lemma 9.2.4 of Ghosh et al. (1997) and the conditions of Lemma Equation 7, we conclude that $\mathrm{E}_{11}=O\left(\sqrt{\epsilon} / n_{c}\right)$. Similarly, using the Cauchy-Schwarz inequality, Lemma 6 and the conditions of Lemma Equation 7, we have $\mathrm{E}_{12}=O\left(\sqrt{\epsilon} / n_{c}\right)$. Therefore, $\mathrm{E}_{1}=O\left(\sqrt{\epsilon} / n_{c}\right)$. Following the same arguments as above, one can show that $\mathrm{E}_{2}=O\left(\sqrt{\epsilon} / n_{c}\right)$. Hence, Lemma 7 is proven.

Proof of Part 1 of Theorem 1: The definition of the stopping rule $N_{c}$ in Equation 25 yields

$$
\sqrt{\frac{A}{c}} V_{N_{c}} \leq N_{c} \leq m I\left(N_{c}=m\right)+\sqrt{\frac{A}{c}}\left(V_{N_{c}-1}+\left(N_{c}-1\right)^{-\gamma}\right)
$$

where $I(\cdot)$ is the indicator function. Because $N_{c} \rightarrow \infty$ asymptotically as $c \downarrow 0$, $P\left(N_{c}=m\right)=0$ and $V_{n} \rightarrow \xi$ asymptotically as $n \rightarrow \infty$, by theorem 2.1 of Gut (2009), $V_{N_{c}} \rightarrow \xi$ asymptotically. Hence, dividing all sides of Equation 44 by $n_{c}$ and letting $c \downarrow 0$, we prove $N_{c} / n_{c} \rightarrow 1$ asymptotically as $c \downarrow 0$.

Because $N_{c} \geq m$ asymptotically and $n_{c} \geq 1$, dividing Equation 44 by $n_{c}$ yields

$$
N_{c} / n_{c} \leq m I\left(N_{c}=m\right) / n_{c}+\frac{1}{\xi}\left(\sup _{c>0} V_{N_{c}-1}+(m-1)^{-\gamma}\right) \quad \text { almost surely }
$$

where $E\left(\sup _{c>0} V_{N_{c}-1}\right)<\infty$ by Lemma 5 . Because $N_{c} / n_{c} \rightarrow 1$ asymptotically as $c \downarrow 0$, by the dominated convergence theorem and $P\left(N_{c}=m\right)=0$, we conclude that $\lim _{c \downarrow 0} \mathrm{E}\left(N_{c} / n_{c}\right)=1$.

Proof of Part 2 of Theorem 1: We need to show

$\lim _{c \downarrow 0} R_{N_{c}}(\kappa) / R_{n_{c}}^{*}(\kappa)=\lim _{c \downarrow 0}\left(A / 2 c n_{c}\right) \mathrm{E}\left[\left(k_{N_{c}}-\kappa\right)^{2}\right]+\frac{1}{2} \lim _{c \downarrow 0} \mathrm{E}\left[N_{c} / n_{c}\right]=1$. Thus, it is enough to show that $\lim _{c \rightarrow 0}\left(A / c n_{c}\right) \mathrm{E}\left[\left(k_{N_{c}}-\kappa\right)^{2}\right]=1$, that is, $\lim _{c \downarrow 0} n_{c} \mathrm{E}\left[\left(k_{N_{c}}-\kappa\right)^{2}\right]=\xi^{2}$. Because we know that $n_{c} \mathrm{E}\left[\left(k_{n_{c}}-\kappa\right)^{2}\right]=\xi^{2}$, it is sufficient to show that

$$
\lim _{c \downarrow 0} n_{c}\left\{\mathrm{E}\left[\left(k_{N_{c}}-\kappa\right)^{2}-\left(k_{n_{c}}-\kappa\right)^{2}\right]\right\}=0 .
$$


Let $\mathrm{E}_{1}=\mathrm{E}\left[\left(k_{N_{c}}-\kappa\right)^{2} I\left(N_{c} \leq n_{2 c}\right)\right]$. By Equation 26, Lemma 3, and Lemma 4, we have

$$
\begin{aligned}
n_{c} \mathrm{E}_{1} & \leq \mathrm{E}\left[\max _{n_{1 c} \leq n \leq n_{2 c}}\left(k_{n}-\kappa\right)^{2} I\left(N_{c} \leq n_{2 c}\right)\right] \\
& \leq n_{c}\left\{\mathrm{E}\left[\max _{n_{1 c} \leq n \leq n_{2 c}}\left(k_{n}-\kappa\right)^{4}\right] P\left(N_{c} \leq n_{2 c}\right)\right\}^{\frac{1}{2}}=O\left(c^{h}\right),
\end{aligned}
$$

where $h=(1-2 \gamma) /(4+4 \gamma)>0$ using $\gamma \in\left(0, \frac{1}{2}\right)$. Here, we assume that $\mathrm{E}\left[X_{1}^{16}\right]$ and $\mathrm{E}\left[X_{1}^{-16 \alpha}\right]$ exist for $\alpha>1$. Following the same arguments as in Lemma 6 , we can show that $\mathrm{E}\left[k_{n_{c}}-\kappa\right]^{4}=O\left(n_{c}^{-2}\right)$ provided $\mathrm{E}\left[X_{1}^{16}\right]$ and $\mathrm{E}\left[X_{1}^{-16 \alpha}\right]$ exist for $\alpha>1$. Let $\mathrm{E}_{2}=\mathrm{E}\left[\left(k_{n_{c}}-\kappa\right)^{2} I\left(N_{c} \leq n_{2 c}\right)\right]$. By the Cauchy-Schwarz inequality, and Lemma 3, we have

$$
n_{c} \mathrm{E}_{1} \leq n_{c}\left\{\mathrm{E}\left[\left(k_{n_{c}}-\kappa\right)^{4}\right] P\left(N_{c} \leq n_{2 c}\right)\right\}^{\frac{1}{2}}=O\left(c^{\frac{1}{1+\gamma}}\right)
$$

provided $\mathrm{E}\left[X_{1}^{16}\right]$ and $\mathrm{E}\left[X_{1}^{-24}\right]$ exist. Therefore, combining Equation 47 and Equation 48, we have

$$
\lim _{c \downarrow 0} n_{c} \mathrm{E}\left[\left\{\left(k_{N_{c}}-\kappa\right)^{2}-\left(k_{n_{c}}-\kappa\right)^{2}\right\} I\left(N_{c} \leq n_{2 c}\right)\right]=0
$$

Using the same arguments as in Lemma 4, one can show that $\mathrm{E}\left[\max _{n \geq n_{3 c}}\left(k_{n}-\kappa\right)^{4}\right]=O\left(n_{3 c}^{-2}\right)$ provided $\mathrm{E}\left[X_{1}^{16}\right]$ and $\mathrm{E}\left[X_{1}^{-16 \alpha}\right]$ exist for $\alpha>1$. Let $\mathrm{E}_{3}=\mathrm{E}\left[\left(k_{N_{c}}-\kappa\right)^{2} I\left(N_{c} \geq n_{3 c}\right)\right]$. The Cauchy-Schwarz inequality and Lemma 3 yield

$$
n_{c} \mathrm{E}_{3} \leq n_{c}\left\{\mathrm{E}\left[\max _{n \geq n_{3 c}}\left(k_{n}-\kappa\right)^{4}\right] P\left(N_{c} \geq n_{3 c}\right)\right\}^{\frac{1}{2}}=O\left(c^{h}\right)
$$

Following the same approach as in Equation $48, n_{c} \mathrm{E}\left[\left(k_{n_{c}}-\kappa\right)^{2} I\left(N_{c} \geq n_{3 c}\right)\right] \leq O\left(c^{\frac{1}{1+\gamma}}\right)$. Thus,

$$
\lim _{c \downarrow 0} n_{c} \mathrm{E}\left[\left\{\left(k_{N_{c}}-\kappa\right)^{2}-\left(k_{n_{c}}-\kappa\right)^{2}\right\} I\left(N_{c} \geq n_{3 c}\right)\right]=0 .
$$


Hence, it remains to prove that

$$
\lim _{c \downarrow 0} n_{c} \mathrm{E}\left[\left\{\left(k_{N_{c}}-\kappa\right)^{2}-\left(k_{n_{c}}-\kappa\right)^{2}\right\} I\left(n_{2 c} \leq N_{c} \leq n_{3 c}\right)\right]=0 .
$$

Let $W=\left\{\left(k_{N_{c}}-\kappa\right)^{2}-\left(k_{n_{c}}-\kappa\right)^{2}\right\} I\left(n_{2 c} \leq N_{c} \leq n_{3 c}\right)$. Note that

$$
\begin{aligned}
W & =\left\{\left(k_{N_{c}}-\kappa\right)+\left(k_{n_{c}}-\kappa\right)\right\}\left(k_{N_{c}}-k_{n_{c}}\right) I\left(n_{2 c} \leq N_{c} \leq n_{3 c}\right) \\
& \leq 2\left\{\max _{n_{2 c} \leq n \leq n_{3 c}}\left|k_{n}-\kappa\right|\right\}\left\{\max _{n_{2 c} \leq n \leq n_{3 c}}\left|k_{n}-k_{n_{c}}\right|\right\} I\left(n_{2 c} \leq N_{c} \leq n_{3 c}\right) .
\end{aligned}
$$

Using the Cauchy-Schwarz inequality, Lemma 7, and proceeding along the lines of Lemma 4, we can write,

$$
\begin{aligned}
n_{c} \mathrm{E}[W] & \leq 2 n_{c}\left\{\mathrm{E}\left[\max _{n_{2 c} \leq n \leq n_{3 c}}\left(k_{n}-\kappa\right)^{2}\right] \mathrm{E}\left[\max _{n_{2 c} \leq n \leq n_{3 c}}\left(k_{n}-k_{n_{c}}\right)^{2}\right]\right\}^{\frac{1}{2}} \\
& \leq 2 n_{c}\left\{O\left(n_{c}^{-1}\right) O\left(\frac{\sqrt{\epsilon}}{n_{c}}\right)\right\}^{\frac{1}{2}}=O\left(\epsilon^{1 / 4}\right) .
\end{aligned}
$$

Because Equation 54 is true for any $\epsilon \in(0,1)$, taking the limit on both sides of Equation 54 as $\epsilon \rightarrow 0$, Equation 52 is proven. Hence, the proof of Theorem 1 is complete.

A Simulation Study Showing the Neglibility of the Remainder Term, $\eta$, of MSE of $k_{n}$

We now provide the results of a simulation study that gives the absolute difference between the estimated mean square error $\left(\widehat{M S E}_{n}\right)$ and the asymptotic mean square error $\xi^{2} / n$. In each replication, a sample of $n=(20,100,500)$ observations were randomly drawn from four different distributions with several parameter choices. In all cases, the number of replications used was 5,000. The third, fourth, and fifth columns of the Table 5 show that ignoring $\eta$ has a negligible effect on the value of the mean square error. 


\section{References}

Chattopadhyay, B., \& Kelley, K. (in press). Estimation of the coefficient of variation with minimum risk: A sequential method for minimizing sampling error and study cost. Multivariate Behavioral Research, X, X-X.

De, S. K., \& Chattopadhyay, B. (2015, 27th March). Minimum risk point estimation of gini index. Retrieved from http://arxiv.org/abs/1503.08148

Doob, J. L. (1953). Stochastic processes. New York, NY: John Wiley \& Sons.

Ghosh, M., Mukhopadhyay, N., \& Sen, P. K. (1997). Sequential estimation. New York, NY: John Wiley \& Sons.

Gut, A. (2009). Stopped random walks: Limit theorems and applications. Cambridge, England: Springer.

Jeffreys, H., \& Jeffreys, B. (1988). The lipschitz condition, § 1.15 in methods of mathematical physics. Cambridge, England: Cambridge University Press.

Lee, A. J. (1990). U-statistics: Theory and practice. New York, NY: CRC Press.

Loève, M. (1963). Probability theory. Princeton, NJ: Van Nostrand.

Sen, P. K. (1981). Sequential nonparametrics: Invariance principles and statistical inference. New York, NY: John Wiley \& Sons.

Sen, P. K., \& Ghosh, M. (1981). Sequential point estimation of estimable parameters based on u-statistics. Sankhyā: The Indian Journal of Statistics, Series A, 331-344. Retrieved from http://www.jstor.org/stable/25050282 


\begin{abstract}
Author Note
Both authors contributed equally and authorship is alphabetical. Correspondence concerning this article should be addressed to Bhargab Chattopadhyay, Department of Mathematical Sciences, 800 West Campbell Rd, FO 2.402A, University of Texas at Dallas, Richardson, TX 75080 (email: bhargab@utdallas.edu), or Ken Kelley, Department of Management, Mendoza College of Business, University of Notre Dame, Notre Dame, IN 46556 (email: kkelley@nd.edu).
\end{abstract}




\section{Footnotes}

${ }^{1} \mathrm{~A}$ sequence $W_{n}$ is a submartingale if $\mathrm{E}\left[W_{t} \mid\left\{W_{\tau}: \tau \leq s\right\}\right] \geq W_{s}$ for all $s \leq t$. 
Table 1. Absolute Difference Between Estimated MSE $\left(\widehat{M S E}_{n}\right)$ and the Theoretical Asymptotic MSE $\left(\xi^{2} / n\right)$

\begin{tabular}{|c|c|c|c|c|}
\hline \multirow[t]{2}{*}{ Distribution } & \multirow[t]{2}{*}{$\kappa$} & \multicolumn{3}{|c|}{$\left|\widehat{M S E}_{n}-\xi^{2} / n\right|$} \\
\hline & & $n=20$ & $n=100$ & $n=500$ \\
\hline $\begin{array}{c}\text { Gamma } \\
(\text { shape }=25, \text { scale }=0.6)\end{array}$ & 0.2 & $9.2014 \times 10^{-4}$ & $1.8457 \times 10^{-4}$ & $3.6927 \times 10^{-5}$ \\
\hline $\begin{array}{c}\text { Gamma } \\
(\text { shape }=6.25, \text { scale }=0.6)\end{array}$ & 0.4 & $2.9543 \times 10^{-3}$ & $6.0414 \times 10^{-4}$ & $1.2098 \times 10^{-4}$ \\
\hline $\begin{array}{c}\text { Gamma } \\
(\text { shape }=2.7778, \text { scale }=0.6)\end{array}$ & 0.6 & $5.7837 \times 10^{-3}$ & $1.1995 \times 10^{-3}$ & $2.3983 \times 10^{-4}$ \\
\hline $\begin{array}{c}\text { Gamma } \\
(\text { shape }=1.5625, \text { scale }=0.6)\end{array}$ & 0.8 & $1.4762 \times 10^{-2}$ & $3.0174 \times 10^{-3}$ & $6.0322 \times 10^{-4}$ \\
\hline $\begin{array}{c}\text { Log-normal } \\
(\log \text {-scale }=1, \text { shape }=0.19805)\end{array}$ & 0.2 & $1.5990 \times 10^{-3}$ & $3.2560 \times 10^{-4}$ & $6.5275 \times 10^{-5}$ \\
\hline $\begin{array}{c}\text { Log-normal } \\
(\log \text {-scale }=1, \text { shape }=0.304)\end{array}$ & 0.4 & $1.5990 \times 10^{-3}$ & $3.2454 \times 10^{-4}$ & $6.5000 \times 10^{-5}$ \\
\hline $\begin{array}{c}\text { Log-normal } \\
(\log \text {-scale }=1, \text { shape }=0.5545)\end{array}$ & 0.6 & $5.5603 \times 10^{-3}$ & $1.2751 \times 10^{-3}$ & $2.6437 \times 10^{-4}$ \\
\hline $\begin{array}{c}\text { Log-normal } \\
(\log \text {-scale }=1, \text { shape }=0.703345)\end{array}$ & 0.8 & $5.0614 \times 10^{-2}$ & $1.0623 \times 10^{-2}$ & $2.1784 \times 10^{-3}$ \\
\hline $\begin{array}{c}\text { Folded-normal } \\
(\text { location }=10, \text { scale }=2)\end{array}$ & 0.2 & $1.0749 \times 10^{-3}$ & $2.1593 \times 10^{-4}$ & $4.3199 \times 10^{-5}$ \\
\hline $\begin{array}{c}\text { Folded-normal } \\
(\text { location }=10, \text { scale }=4.052)\end{array}$ & 0.4 & $4.9931 \times 10^{-3}$ & $9.9890 \times 10^{-4}$ & $1.997 \times 10^{-4}$ \\
\hline $\begin{array}{c}\text { Folded-normal } \\
(\text { location }=10, \text { scale }=7.0735)\end{array}$ & 0.6 & $1.0261 \times 10^{-2}$ & $2.0404 \times 10^{-3}$ & $4.0795 \times 10^{-4}$ \\
\hline $\begin{array}{c}\text { Normal } \\
(\text { Mean }=10, \mathrm{SD}=2)\end{array}$ & 0.2 & $1.0768 \times 10^{-3}$ & $2.1568 \times 10^{-4}$ & $4.3190 \times 10^{-5}$ \\
\hline $\begin{array}{c}\text { Normal } \\
(\mathrm{Mean}=10, \mathrm{SD}=4)\end{array}$ & 0.4 & $5.2779 \times 10^{-3}$ & $1.0560 \times 10^{-3}$ & $2.1118 \times 10^{-4}$ \\
\hline $\begin{array}{c}\text { Normal } \\
(\text { Mean }=10, \mathrm{SD}=6)\end{array}$ & 0.6 & $1.5514 \times 10^{-2}$ & $3.0955 \times 10^{-3}$ & $6.1918 \times 10^{-4}$ \\
\hline $\begin{array}{c}\text { Normal } \\
(\text { Mean }=10, \mathrm{SD}=8)\end{array}$ & 0.8 & $3.6132 \times 10^{-2}$ & $7.2953 \times 10^{-3}$ & $1.4592 \times 10^{-3}$ \\
\hline $\begin{array}{c}\text { Weibull } \\
(\text { shape }=5.7973, \text { scale }=1)\end{array}$ & 0.2 & $4.4499 \times 10^{-4}$ & $4.0008 \times 10^{-5}$ & $3.4786 \times 10^{-6}$ \\
\hline $\begin{array}{c}\text { Weibull } \\
(\text { shape }=2.6956223, \text { scale }=1)\end{array}$ & 0.4 & $1.4018 \times 10^{-3}$ & $1.1774 \times 10^{-4}$ & $1.0636 \times 10^{-5}$ \\
\hline $\begin{array}{c}\text { Weibull } \\
(\text { shape }=1.717082, \text { scale }=1)\end{array}$ & 0.6 & $2.3447 \times 10^{-3}$ & $2.0318 \times 10^{-4}$ & $1.8057 \times 10^{-5}$ \\
\hline $\begin{array}{c}\text { Weibull } \\
(\text { shape }=1.258237, \text { scale }=1)\end{array}$ & 0.8 & $6.3154 \times 10^{-3}$ & $8.5099 \times 10^{-4}$ & $1.0147 \times 10^{-4}$ \\
\hline
\end{tabular}

Note: $\kappa$ is the population coefficient of variation; $n$ is the sample size; $\widehat{M S E}_{n}$ is the estimated mean square error (MSE); $\xi^{2} / n$ the theoretical Asymptotic MSE; tabled values are based on 5,000 replications of a Monte Carlo simulation study. 